\title{
High interstitial fluid pressure promotes tumor cell proliferation and invasion in oral squamous cell carcinoma
}

\author{
TAO YU ${ }^{1,2^{*}}$, KUN LIU $^{1 *}$, YINGYING WU $^{3}$, JINCHUAN FAN $^{1}$, JIANCHAO CHEN $^{1}$, \\ CHAO LI ${ }^{1}$, GUIQUAN ZHU ${ }^{1}$, ZHAOHUI WANG ${ }^{1}$ and LONGJIANG $\mathrm{LI}^{2,3}$ \\ ${ }^{1}$ Department of Head and Neck Oncology Surgery, Sichuan Cancer Hospital; \\ ${ }^{2}$ Department of Head and Neck Oncology Surgery, ${ }^{3}$ State Key Laboratory of Oral Diseases, \\ West China College of Stomatology, Sichuan University, Chengdu, Sichuan 610041, P.R. China
}

Received June 13, 2013; Accepted September 10, 2013

DOI: $10.3892 /$ ijmm.2013.1496

\begin{abstract}
It has been shown that interstitial fluid pressure (IFP) is elevated in many solid tumors. The elevated IFP in tumors is responsible, at least in part, for the poor blood supply, inadequate delivery of therapeutic agents to solid tumors and poor treatment response in patients. The present study was carried out to examine alterations in malignant phenotypes in oral squamous cell carcinoma cells subjected to conditions mimicking IFP and to identify the relevant molecular mechanisms. We investigated tumor cell proliferation and invasion using SCC-4 and SCC-9 cells subjected to an increased extracellular pressure of 0,15 and $30 \mathrm{mmHg}$ in vitro. The results revealed that the increased IFP resulted in a marked increase in cancer cell proliferation, survival and invasion in vitro and altered the expression of $>1,800$ genes involved in invasion and metastasis, the heat shock pathway, the p38 and JNK signaling pathway, apoptosis and the cell growth and differentiation signaling pathway. These results suggest the important potential clinical application of measuring IFP, which can be used as a generic marker of prognosis and response to therapy.
\end{abstract}

\section{Introduction}

Tumors are characterized by high geometric and viscous resistance to blood flow, high microvascular hydrostatic pressure, low resistance to transcapillary fluid flow, high resistance to inter-

Correspondence to: Professor Longjiang Li, Department of Head and Neck Oncology Surgery, West China College of Stomatology, Sichuan University, No. 14, Sec. 3, Renminnan Road, Chengdu, Sichuan 610041, P.R. China

E-mail: muzili63@126.com

Professor Zhaohui Wang, Department of Head and Neck Oncology Surgery, Sichuan Cancer Hospital, No. 55, Sec. 4, Renminnan Road, Chengdu, Sichuan 610041, P.R. China

E-mail: zhaohuiwang512@163.com

${ }^{*}$ Contributed equally

Key words: interstitial fluid pressure, oral squamous cell carcinoma, tumor progression, microarray stitial fluid flow, impaired lymphatic drainage and an abnormal extracellular matrix $(1,2)$. Excess fluid leaks from the vasculature into the interstitium, where it accumulates and distends the elastic extracellular matrix (ECM), elevating interstitial fluid pressure (IFP). Solid tumors present with an IFP that is elevated above that of normal tissues. A number of studies have shown that solid malignant tumors have IFP values of 5 to $40 \mathrm{mmHg}$, whereas the IFP of most normal tissues ranges from -3 to $+3 \mathrm{mmHg}(3-6)$. Gutmann et al (3) measured IFP in squamous cell carcinomas of the head and neck region in humans using the wick-in-needle technique and identified that the IFP ranged from 4 to $33 \mathrm{mmHg}$. The increase in IFP leads to a positive pressure gradient, which is a driving force for a convective transport back into the capillaries or to adjacent regions with low IFP. Such convective forces inhibit the transfer of drugs to the tumor interstitium and facilitate tumor cell intravasation into the vascular or lymphatic circulation, and hence promote metastasis (7). Furthermore, there are clinical data showing that tumor IFP correlates with response to treatment, with strong evidence for high IFP as a poor prognostic indicator in patients with cervical cancer treated with radiotherapy $(6,8)$. Patients are significantly more likely to develop distant recurrence if they present with a tumor IFP value above the group median $(19 \mathrm{mmHg})$, which suggests a role for IFP in metastatic spread (9).

Although local oral squamous cell carcinoma (OSCC) can be effectively controlled by surgical excision and radiotherapy, metastasis to the lymph nodes and distant organs significantly decreases the survival rate (10). Metastasis is the final step in solid tumor progression and is the most common cause of mortality in cancer patients (11). How tumor cells become metastatic is largely unknown. However, several mechanisms have been suggested, including the possibility that tumors with a high IFP may have a high rate of cell proliferation and metastasis (2). It was widely believed that metastatic cells are rare and evolve during thelate stages of tumor progression from a series of changes in gene expression that enable the cells to progress through the sequential steps that finally result in growth in distant organ microenvironments.

Experimental studies attempting to relate the outcome of cancer treatment to IFP are rare. Thus, the mechanisms linking an elevated IFP to poor survival rates in patients with OSCC are not yet well understood. In this study, we subjected SCC-4 
and SCC-9 OSCC cells to conditions mimicking IFP in vitro. The cells were incubated for $24 \mathrm{~h}$ under 0,15 and $30 \mathrm{mmHg}$ increased extracellular pressure and we then investigated the alterations in malignant phenotypes and the relevant molecular mechanisms. Furthermore, we identified that an elevated extracellular pressure significantly promotes cancer cell proliferation and invasion by altering the expression of $>1,800$ genes, which are involved in invasion and metastasis, the heat shock pathway, the p38 and JNK signaling pathway, apoptosis and the cell growth and differentiation signaling pathway using microarray analysis.

\section{Materials and methods}

Cell culture and reagents. SCC-4 and SCC-9 human tongue squamous cell carcinoma cell lines were obtained from ATCC. The cells were cultured at $37^{\circ} \mathrm{C}$ in $5 \% \mathrm{CO}_{2}$ in RPMI- 1640 containing $10 \%$ fetal bovine serum (FBS; Gibco Life Technologies, Carlsbad, CA, USA), $2 \mathrm{mM}$ glutamine, $1 \mathrm{mM}$ sodium pyruvate, $10 \mathrm{mM}$ HEPES, $100 \mathrm{U} / \mathrm{ml}$ penicillin $\mathrm{G}$ and $100 \mathrm{mg} / \mathrm{ml}$ streptomycin (Sigma, USA). The cell proliferation and cytotoxicity assay kit was from Dojindo Laboratories (Kumamoto, Japan). The real-time PCR primers were synthesized commercially (Takara, Kyoto, Japan). The Invasion Assay kit was from Millipore (Billerica, MA, USA). All tissue culture media and sera were from Gibco Life Technologies. Peroxidase-labeled anti-rabbit antibody and enhanced chemiluminescence (ECL) system were from Millipore.

Pressure regulation. The pressure system used in the present study was custom designed (Sichuan University, Chengdu, China) to expose cultured tumor cells to well-defined stable pressure regimes appropriate for modeling IFP levels in tumors. The cells were placed in an airtight stainless steel box with an inlet valve through which the air flowed into the box and an air pump was connected to this box. There is an absolute pressure sensor (measuring range $30-120 \mathrm{kPa}$; VTI Technologies, Finland) in the box, which can promptly sense and respond to variations ins pressure. Control and data acquisition programs utilized a pre-calibrated differential pressure transducer to acquire pressure data (needed to control the opening and closing of the appropriate solenoid valves). The pressure box was warmed and maintained at a temperature of $37^{\circ} \mathrm{C}$ for at least $1 \mathrm{~h}$ prior to each experiment to prevent pressure fluctuations due to temperature shifts of the pressurizing gas. Temperature was maintained within $\pm 2^{\circ} \mathrm{C}$ and pressure within $\pm 1.5 \mathrm{mmHg}$. The whole experimental facility apart from the electronic control panel was placed in a $5 \% \mathrm{CO}_{2}$ incubator. The desired pressure was achieved within $1 \mathrm{~min}$ for the experiments with the cells. Cells subjected to pressure were placed inside this pressure box for $24 \mathrm{~h}$ with the pressure set at 15 and $30 \mathrm{mmHg}$ above atmospheric pressure. The blank group cells were incubated at $37^{\circ} \mathrm{C}$ in $5 \% \mathrm{CO}_{2}$ under ambient pressure for the same amount of time (Fig. 1).

Cell proliferation assay. Cell proliferation assays were performed using the Cell Counting kit-8 (CCK-8; Dojindo Laboratories). Cells were plated in 96-well plates at $1 \times 10^{4}$ cells/well and incubated for five days. Each day, at a certain time, $10 \mu 1$ of CCK- 8 solution were added to each well and the cells were incubated
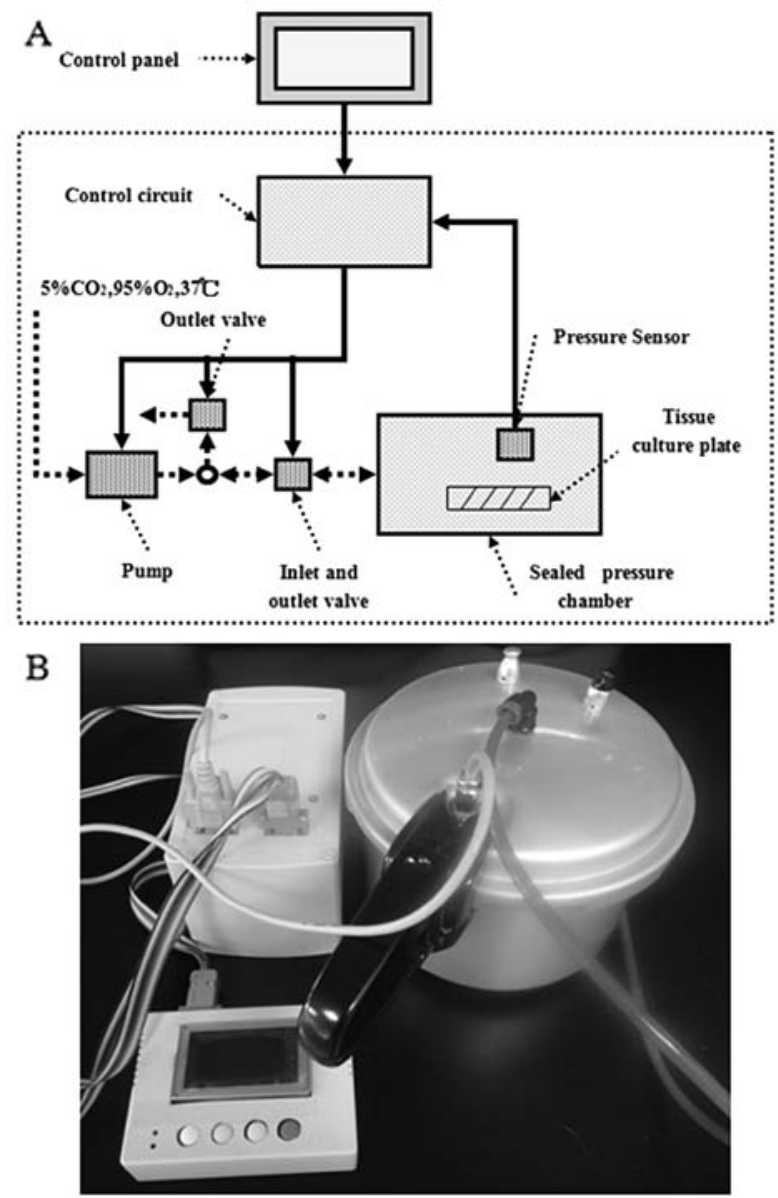

Figure 1. The cyclic pressure system and the interstitial fluid pressure (IFP) instrument used in the present study. (A) Schematic diagram of the conditions used to mimic increased IFP. (B) Image of the conditions used to mimic increased IFP. Briefly, during the experiments, the cancer cells placed in the sealed, $37^{\circ} \mathrm{C}, 5 \% \mathrm{CO}_{2}$, humidified pressure chamber were exposed to cyclic pressure regimes characterized by minimum and maximum pressures $(0$ and $40 \mathrm{mmHg}$ above atmospheric) for $24 \mathrm{~h}$; the cells placed in the unsealed control chambers served as the experimental controls.

for a further $2 \mathrm{~h}$. The optical density (OD) value was obtained by the differences in absorbance at a wavelength of $450 \mathrm{~nm}$ using a microplate reader (Varioskan Flash 3001; Thermo Scientific, Waltham, MA, USA). The amount of formazan dye, generated by the activities of dehydrogenases in the cells, is directly proportional to the number of living cells. In addition, we used plate colony formation assay to evaluate the colony forming ability of the tumor cells. The tumor cells were cultured at 500 cells $/ 5 \mathrm{ml}$ with RPMI-1640 medium and 10\% FBS in a 6-well plate. After ten days in culture, the cells were fixed with methanol for $10 \mathrm{~min}$ and stained with $1 \%$ crystal violet solution for $20 \mathrm{~min}$ to visualize the colonies for cell counting.

Cell invasion assay. The cell invasion assay was performed using the QCM ${ }^{\mathrm{TM}}$ 12-well Invasion Assay kit (Chemicon International, Inc., Temecula, CA, USA). This cell invasion assay is performed in an invasion chamber, based on the Boyden chamber principle. This kit contains 12 inserts; each insert contains an $8-\mu \mathrm{m}$ pore size polycarbonate membrane coated with a thin layer of ECMatrix $^{\mathrm{TM}}$. Briefly, the cells were resuspended in serum-free RPMI-1640, and $2 \times 10^{5}$ cells were added to the interior of the 
Table I. Genes and primer sequences used for quantitative RT-PCR.

\begin{tabular}{|c|c|c|}
\hline Genes & Forward primer $\left(5^{\prime}-3^{\prime}\right)$ & Reverse primer $\left(5^{\prime}-3^{\prime}\right)$ \\
\hline HSPA4 & GGAGGAACCACATGTTGAAG & TGGATCCAGCTTGAGAGGTC \\
\hline VEGFC & AACCTCCATGTGTGTCCGTC & TGGCAAAACTGATTGTTACTGG \\
\hline VEGFR3 & CTACAAAGACCCCGACTACG & CGTAGTCGGGGTCTTTGTAG \\
\hline KISS1 & CTTGGCAGCTACTGCTTTTC & GTAGCAGCTGGCTTCCTCTC \\
\hline TWIST1 & GGCTCAGCTACGCCTTCTC & CTAGTGGGACGCGGACAT \\
\hline VEGFD & GTATGGACTCTCGCTCAGCAT & AGGCTCTCTTCATTGCAACAG \\
\hline MAPK8 & ACGCCTTATGTAGTGACTCGCTACT & TTGTAGCCCATGCCAAGGA \\
\hline PAI2 & GCATCCACTGGCTTGGAA & GGGAATGTAGACCACAACATCAT \\
\hline FOS & TCGGGCTTCAACGCAGACTACG & TGACCGTGGGAATGAAGTTGGC \\
\hline HIF1A & CATAAAGTCTGCAACATGGAAGGT & ATTTGATGGGTGAGGAATGGGTT \\
\hline CDKN1A & CTGCCCAAGCTCTACCTTCC & CAGGTCCACATGGTCTTCCT \\
\hline BAX & GCCCTTTTGCTTCAGGGTTT & TCCAATGTCCAGCCCATGAT \\
\hline FOSL1 & ATCCCCGACCTCTGACCTAT & CAAGGCGTTCCTTCTGCTT \\
\hline CD44 & CCTCCAGTGAAAGGAGCAGCAC & GTGTCTTGGTCTCTGGTAGCAGGGAT \\
\hline CCND1 & ACAAGTGTGTCTTACGTGCCACCAC & ACGACAGACAAAGCGTCCCTCAAG \\
\hline STAB 1 & GTTTGTCACTCACACACCCTGT & ATAGCGGCAGTCCAGAAGTATC \\
\hline BAK1 & GAACAGGAGGCTGAAGGGGT & TCAGGCCATGCTGGTAGACG \\
\hline CASP1 & AATGATTGAGAAACTCTTCACTGTGT & CGGGGTACCAAGCCTAGGAAACACAAGGAGA \\
\hline ERBB2 & AGCAGACCCAGTACCTGTCC & AGGGTTGGTCCTTCTATGAGAAT \\
\hline NM23 & ATGGCCAACTGTGAGCGTACC & TTCATAGATCCAGTTCTGAGCACAAGC \\
\hline BCL2 & GACAGAAGATCATGCCGTCC & GGTACCAATGGCACTTCAAG \\
\hline ANKK1 & CGGCTGGCCAAGTTGTCTAA & AGCACCTTCCTGAGTGTCATCA \\
\hline TNFRSF6 & TATGCTTCTTCGTGCAGCAGTT & GCTGCCACACGCTCCTCTAG \\
\hline SMAD2 & CCAGGTCTCTTGATGGTCGT & TATATCCAGGAGGTGGCGTT \\
\hline GAPDH & GAAGGTGAAGGTCGGAGTC & GAAGATGGTGATGGGATTTC \\
\hline
\end{tabular}

inserts, which had been previously rehydrated at room temperature for $30 \mathrm{~min}$. A total of $500 \mu \mathrm{l}$ of RPMI-1640 containing $10 \%$ FBS was added to the lower chamber as a chemoattractant. The cells were incubated for $48 \mathrm{~h}$ at $37^{\circ} \mathrm{C}$ in a $\mathrm{CO}_{2}$ incubator $\left(5 \% \mathrm{CO}_{2}\right)$. Subsequently, the upper surface of the chamber was scraped to remove non-migratory cells. The migrated cells were fixed and stained with $0.1 \%$ crystal violet and photographed under a light microscope (x100). The stained insert was transferred to a clean well containing $500 \mu \mathrm{l}$ of $10 \%$ acetic acid for $15 \mathrm{~min}$ at room temperature. The OD of the stained cells was measured at $560 \mathrm{~nm}$.

Microarray analysis. The 44K Human Genome Oligo Microarray including 44,000 60-mer oligonucleotide probes representing 41,000 unique genes and transcripts was purchased from Agilent Technologies (Palo Alto, CA, USA). In brief, total RNA from the SCC-4 and SCC-9 cells treated with normal ambient pressure, 15 and $30 \mathrm{mmHg}$, were harvested using TRIzol reagent (Invitrogen, Carlsbad, CA, USA) and the RNeasy kit (Qiagen, Hilden, Germany). The amplification and labeling of $500 \mathrm{ng}$ of total RNA was performed according to the protocol provided with the Agilent Quick Amp labeling kit using Cy3. Hybridization was performed for $16 \mathrm{~h}$ at $50^{\circ} \mathrm{C}$. Following hybridization and washing, the processed slides were scanned using the Agilent DNA microarray scanner (part no. G2505B; Agilent Technologies). The resulting text files extracted using Agilent Feature Extraction
Software (version 10.5.1.1) were imported into the Agilent GeneSpring GX software (version 10.0) for further analysis. To identify the genes that were differentially expressed, we performed a fold-change screening between the two groups obtained from the experiment. The threshold we used to screen the upregulated or downregulated genes was a fold-change of $>2$. Hierarchical clustering and a tree diagram were generated using Cluster 3.0 software.

Quantitative reverse transcription PCR ( $q R T-P C R)$. Total RNA was isolated from $1 \times 10^{6}$ cells using TRIzol reagent (Invitrogen). Total RNA was subsequently reverse transcribed into cDNA using the SuperScript First-Strand cDNA Synthesis kit (Invitrogen). qRT-PCR was performed using the SYBR premix Ex Taq ${ }^{\mathrm{TM}}$ II kit (Takara). The specific primers used in this study are listed in Table I. The comparative threshold cycle (CT) method was used to calculate the amplification fold. The GAPDH gene was used as a reference control gene to normalize the expression value of the target genes. Triple replicates were performed for each gene and the average expression value was computed for subsequent analysis. The relative expression level of the genes was calculated using the $2^{-\Delta \Delta \mathrm{Ct}}$ method as previously described (12).

Statistical analysis. Data are expressed as the means \pm standard deviation (SD), when normally distributed. The statistical significance of differences was determined using the Student's 

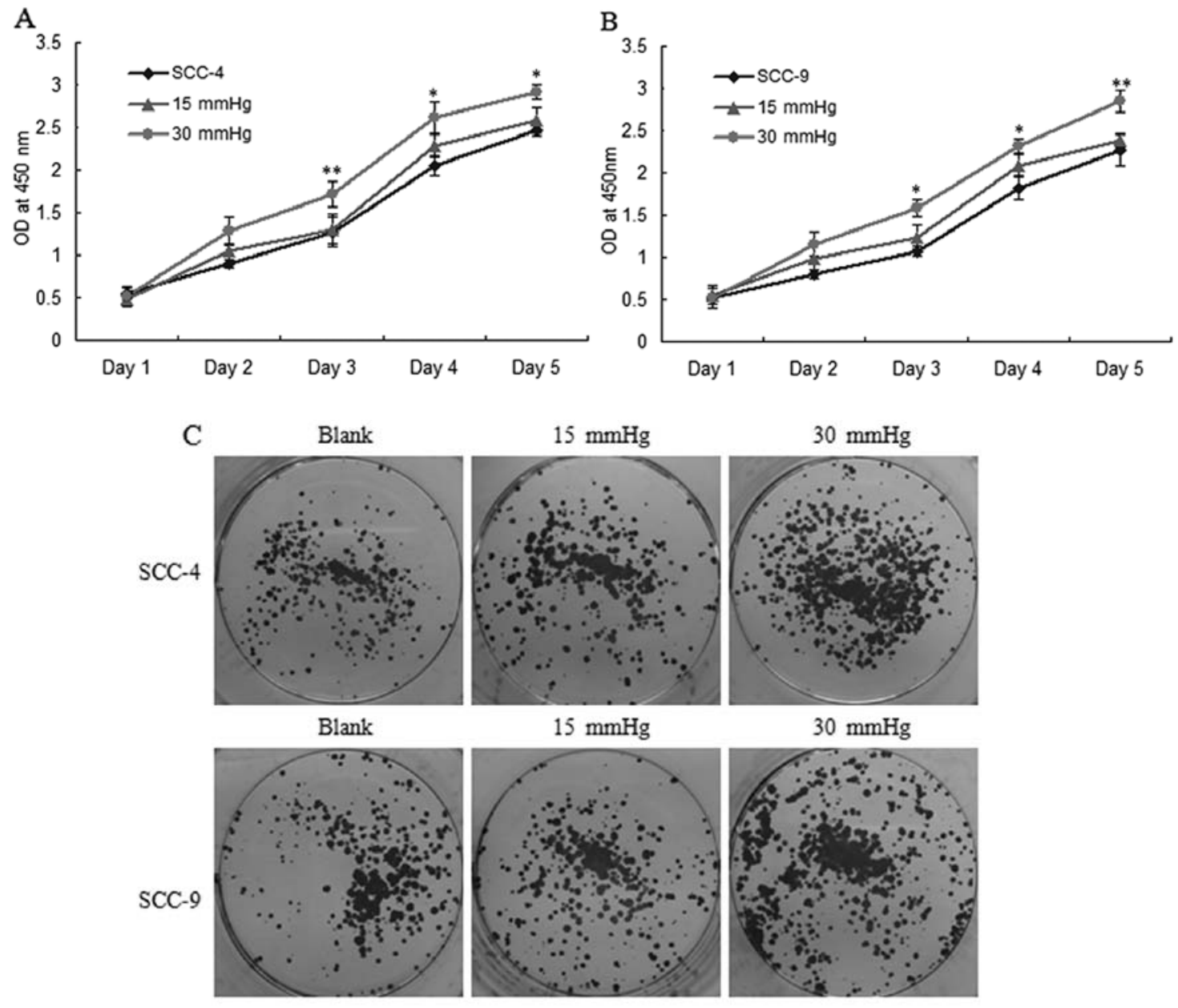

$15 \mathrm{mmHg}$

$30 \mathrm{mmHg}$
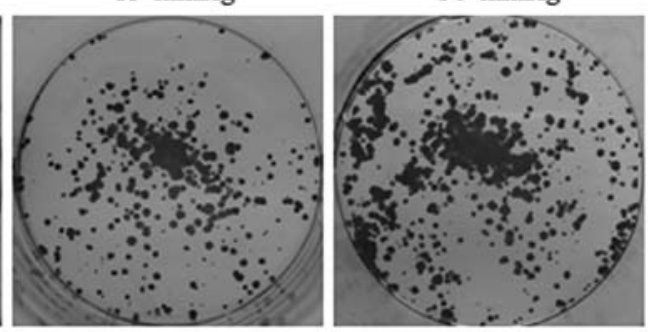

D
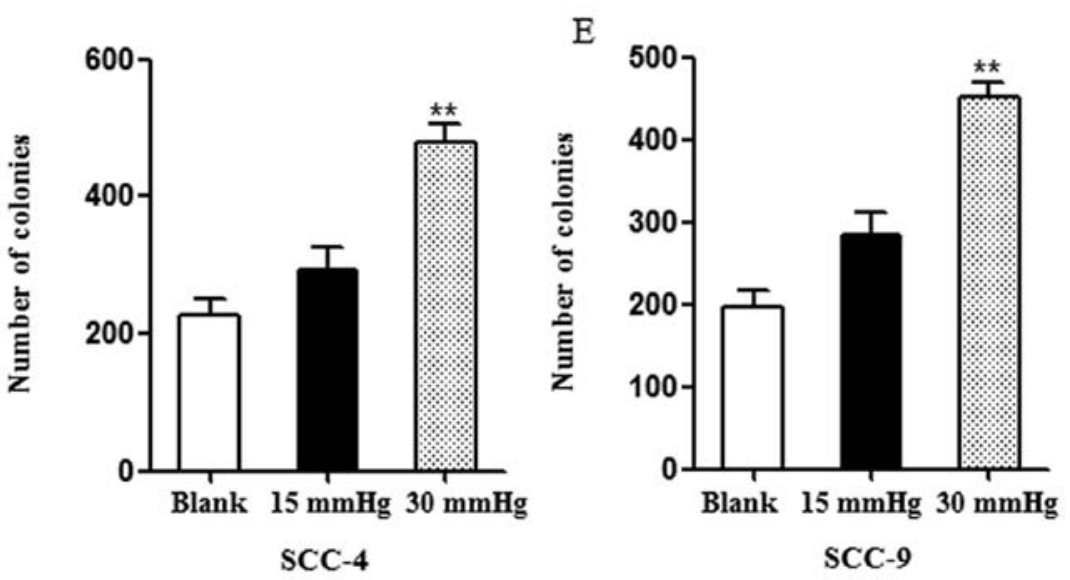

Figure 2. Elevated extracellular pressure increases cell proliferation and the survival of SCC-4 and SCC-9 oral squamous cell carcinoma (OSCC) cells in vitro. (A and B) Following the exposure of SCC-4 and SCC-9 cells with 15 and $30 \mathrm{mmHg}$ pressure for $24 \mathrm{~h}$ in, the number of viable cells was assessed using the Cell Counting Kit-8 (CCK-8) assay. (C) Representative images of plate colony formation in the SCC-4 and SCC-9 cells. (D and E) Quantitative analysis of plate colony formation of SCC-4 and SCC-9 cells.

two-tailed t-test for two groups and one-way ANOVA for multiple groups. A P-value $<0.05$ was considered to indicate a statistically significant difference. All data were analyzed using SPSS 15.0 software.

\section{Results}

Elevated extracellular pressure promotes OSCC cell proliferation and survival in vitro. To detect the effects of extracellular pressure on OSCC cell proliferation and survival, we used the
CCK-8 and plate colony formation assays to assess the effect of extracellular pressure on the proliferation and survival of SCC-4 and SCC-9 cells $(0,15$ and $30 \mathrm{mmHg})$. The results revealed that elevated extracellular pressure significantly increased cancer cell proliferation and colony formation, particularly in the group exposed to $30 \mathrm{mmHg}$ pressure $(\mathrm{P}<0.05)$ (Fig. 2A-E).

Increased extracellular pressure promotes OSCC cell invasion in vitro. The proteolytic degradation of the ECM components is critical for tumor cell invasion. Matrigel-coated Millicell 
A

Blank

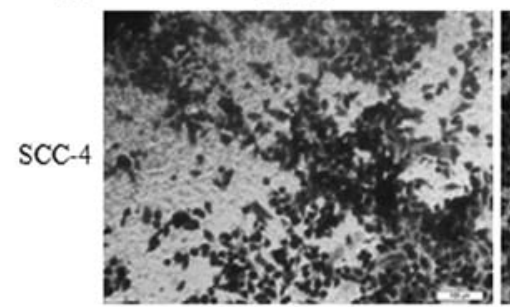

Blank

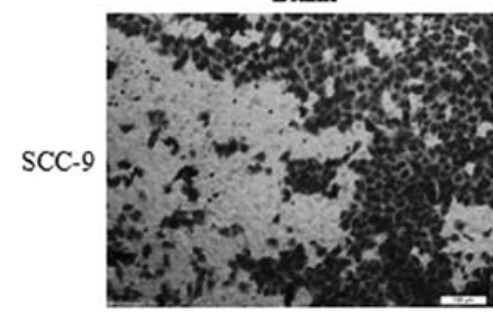

B



$15 \mathrm{mmHg}$

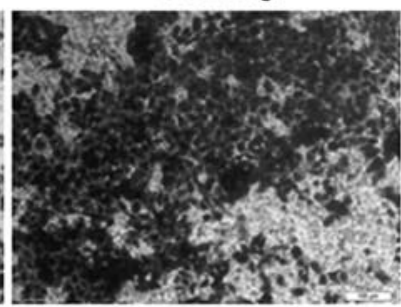

$15 \mathrm{mmHg}$

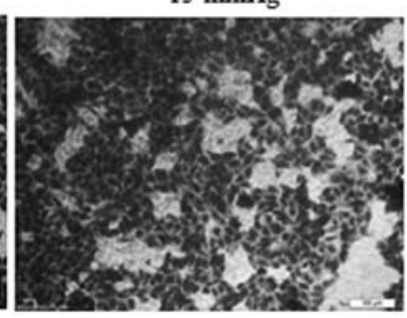

$30 \mathrm{mmHg}$

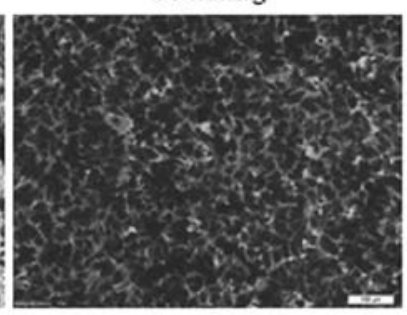

$30 \mathrm{mmHg}$

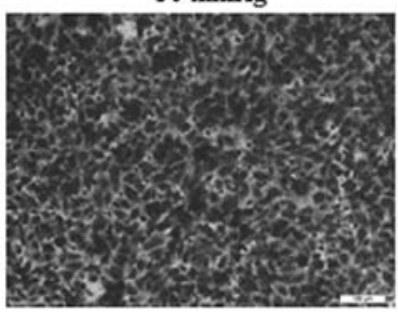

$\mathrm{C}$



Figure 3. Elevated extracellular pressure enhances the cell invasion ability of SCC-4 and SCC-9 oral squamous cell carcinoma (OSCC) cells in vitro. (A) The invading cells on the bottom side of the membrane were stained with $0.1 \%$ crystal violet solution, then the stained inserts were eluted using $10 \%$ acetic acid and the optical density value (OD) was measured at $560 \mathrm{~nm}$. (B and C) Quantitative analysis of the number of invading SCC-4 and SCC-9 cells. Error bars indicate the means $\pm \mathrm{SD} ; \mathrm{n}=3$ experiments; ${ }^{*} \mathrm{P}<0.05 ;{ }^{* *} \mathrm{P}<0.01$.

Chambers were used to determine the influence of extracellular pressure on the invasion capacity of the cancer cells. Quantitative analysis demonstrated that the invasion ability of the cells from the group exposed to $30 \mathrm{mmHg}$ pressure was significantly high, compared with the blank group and the group exposed to $15 \mathrm{mmHg}$ pressure $(\mathrm{P}<0.01)$ (Fig. 3). In addition, $15 \mathrm{mmHg}$ extracellular pressure enhanced the invasion ability of the SCC-4 cells, compared with the blank group $(\mathrm{P}<0.05)$.

Gene expression profile analysis for OSCC cells subjected to elevated extracellular pressure. To further investigate the effects of increased extracellular pressure on downstream targets in OSCC cells, we performed gene expression profiling analysis on the SCC-4 and SCC-9 cells exposed to 0, 15 and $30 \mathrm{mmHg}$ pressure. With the increase in extracellular pressure, unsupervised clustering analysis of 1,827 genes identified two groups of genes (trees 1 and 2) with significantly altered expression levels (by $>2$-fold) in the SCC-4 and SCC-9 cells. Tree 1 included 542 upregulated genes, and tree 2 contained 319 downregulated genes. Functional profiling of these genes revealed that the majority of these genes are involved in inva- sion and metastasis, as well as the heat shock pathway, the p38 and JNK signaling pathway, apoptosis and the cell growth and differentiation signaling pathway (Fig. 4A).

$q R T-P C R$ for tumor-related genes. We then used qRT-PCR to confirm the pressure-dependent expression of over 20 genes identified in the microarray analysis. Apart from the expression of the KISS1 gene in SCC-4 cells, the expression levels of the other genes were consistent with those from microarray analysis (Fig. 4B-C).

\section{Discussion}

Since Young et al 13) firstly identified that IFP is significantly higher in tumors compared with normal tissues, an enhanced IFP has been identified in many solid tumors, and it has been used as an important prognostic factor. It has been shown to be the single best indicator of survival for patients with cancer of the cervix (5). Several studies have demonstrated the correlation between IFP and the malignant characteristics of tumors (14-16). van der Voort van Zyp et al (16) identi- 

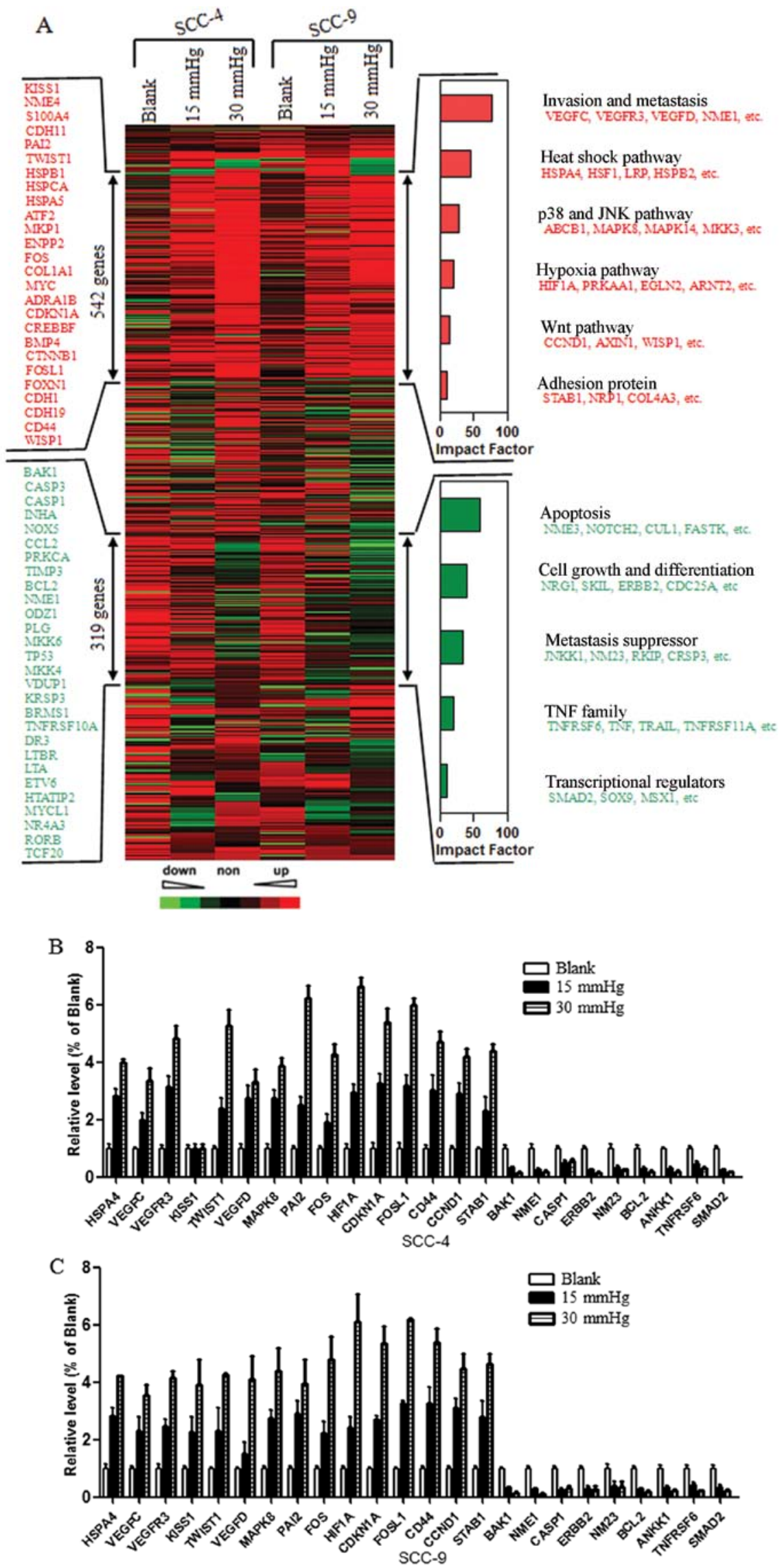

Figure 4. Unsupervised clustering (Cluster 3.0 software) of genes differentially expressed between the control and pressure-exposed SCC-4 and SCC-9 cells. (A) A total of 542 pressure-activated genes and 319 pressure-repressed genes are marked by double-headed arrows. Representative pressure-activated (red) and pressurerepressed genes (green) are either listed vertically or under each molecular pathway. The impact factor strength of pressure-activated (red bars) and -repressed (green bars) genes is shown. (B) Quantitative analysis of the transcript levels of over 20 genes identified by microarray analysis, relative to GAPDH, determined by qRT-PCR and compared with the SCC-4 blank control cells. (C) Quantitative analysis of the transcript levels of over 20 genes identified by microarray analysis, relative to $\beta$-actin, determined by qRT-PCR and compared with the SCC- 9 blank control cells. Error bars indicate the means \pm SD, $n=3$ experiments. 
fied that an increased extracellular pressure of $15 \mathrm{mmHg}$ can stimulate colon cancer cell adhesion to surgical wounds, promoting tumor recurrence. Roh et al (5) found that the IFP increased with the grade (degree of differentiation) of squamous cell carcinoma of the uterine cervix. Although there is no doubt that the IFP is related to tumor size, invasion and metastasis, we wished to determine whether the increased IFP would in turn promote tumor cell proliferation and metastasis. Therefore, in this study, to further investigate the effect of IFP on OSCC cells, we investigated the effects of increased IFP on SCC-4 and SCC-9 cells exposed to conditions mimicking IFP $(0,15$ and $30 \mathrm{mmHg}$ above atmospheric pressure) in vitro. Of note, the proliferation and invasion of SCC-4 cells significantly increased in the group exposed to $30 \mathrm{mmHg}$ pressure in vitro. Furthermore, to our knowledge, this is the first study to demonstrate the effect of a high IFP on OSCC cells. The exposure of OSCC cells to extracellular pressure induces an aggressive cancer phenotype that promotes cancer cell growth and metastasis.

To identify the potential molecular mechanisms responsible for the effects of elevated extracellular pressure on tumor growth and invasion, we used microarray analysis to detect the gene expression profiles in SCC- 4 and SCC-9 cells treated with different pressure values. A total of 1,827 genes with altered expression levels (by $>2$-fold) was identified. A total of 542 genes were upregulated with the increase in pressure in the SCC-4 and SCC-9 cells; these genes were mainly involved in invasion and metastasis, the heat shock pathway, the p38 and JNK signaling pathway and the hypoxia signaling pathway. In addition, 319 genes were found to be downregulated with the elevation in pressure values in the SCC-4 and SCC-9 cells, which were mainly involved in apoptosis, cell growth and differentiation and metastasis. Subsequently, we used qRT-PCR to confirm the pressure-dependent expression of over 20 genes identified in the microarray analysis. Heat shock protein (HSP)A4, also known as HSP70, has been shown to be significantly associated with low differentiation and poor prognosis in a number of malignant tumors (17-19). CCND1, also known as cyclin D1, is overexpressed in several human tumors; its expression is induced by growth factors and occurs at multiple levels, including increased transcription, translation and protein stability (20-22). Cyclin D1 plays an important role in G1 phase transition, which is a kinase-independent function, by sequestering cyclin-dependent kinase (CDK) inhibitors, such as CDKN1A (p21) and CDKN1B (p27) for the efficient activation of CDK2-containing complexes $(23,24)$. Since hypoxia is clearly related to radioresistance, it has been suggested that the abnormal vascularization of tumors leads to increased hypoxia, whereas reperfusion improves the radiosensitivity of tumors $(25,26)$. It has been reported that the hypoxic environment consists of rapidly growing cancer cells that proliferate faster than the vasculature within the environment, creating low oxygen tension in the central and intermediate regions of the tumor mass. This activates the transcriptional factor, hypoxia-inducible factor (HIF-1a), that is known to promote angiogenesis for tumor cell survival and the formation of a central hypoxic region and a normoxic peripheral region; this is possibly regulated by IFP (27-29).

Taken together, to our knowledge, this study demonstrates for the first time the important role of an elevated IFP and the corresponding molecular mechanisms, particularly, the effect of lymphatic metastasis-related gene activation on this process, in promoting OSCC proliferation, as well as invasion and metastasis. Our data suggest the important potential clinical application of measuring IFP; measuring tumor IFP may be a generic marker of prognosis and response to therapy.

\section{Acknowledgements}

This study was supported by the National Natural Science Foundation of China (grant no. 30973345), the Doctoral Fund of Ministry of Education of China (grant no. 20090181110082) and the Fund of the Department of Health of Sichuan Province (grant no. 130231).

\section{References}

1. Cairns R, Papandreou I and Denko N: Overcoming physiologic barriers to cancer treatment by molecularly targeting the tumor microenvironment. Mol Cancer Res 4: 61-70, 2006.

2. Lunt SJ, Fyles A, Hill RP and Milosevic M: Interstitial fluid pressure in tumors: therapeutic barrier and biomarker of angiogenesis. Future Oncol 4: 793-802, 2008

3. Gutmann R, Leunig M, Feyh J, et al: Interstitial hypertension in head and neck tumors in patients: correlation with tumor size. Cancer Res 52: 1993-1995, 1992.

4. Fukumura D and Jain RK: Tumor microenvironment abnormalities: causes, consequences, and strategies to normalize. J Cell Biochem 101: 937-949, 2007.

5. Roh HD, Boucher Y, Kalnicki S, Buchsbaum R, Bloomer WD and Jain RK: Interstitial hypertension in carcinoma of uterine cervix in patients: possible correlation with tumor oxygenation and radiation response. Cancer Res 51: 6695-6698, 1991.

6. Milosevic M, Fyles A, Hedley D, et al: Interstitial fluid pressure predicts survival in patients with cervix cancer independent of clinical prognostic factors and tumor oxygen measurements. Cancer Res 61: 6400-6405, 2001.

7. Rofstad EK, Tunheim SH, Mathiesen B, Graff BA, Halsør EF, Nilsen K and Galappathi K: Pulmonary and lymph node metastasis is associated with primary tumor interstitial fluid pressure in human melanoma xenografts. Cancer Res 62: 661-664, 2002.

8. Fyles A, Milosevic M, Pintilie M, Syed A, Levin W, Manchul L and Hill RP: Long-term performance of interstial fluid pressure and hypoxia as prognostic factors in cervix cancer. Radiother Oncol 80: 132-137, 2006.

9. Lunt SJ, Kalliomaki TM, Brown A, Yang VX, Milosevic M and Hill RP: Interstitial fluid pressure, vascularity and metastasis in ectopic, orthotopic and spontaneous tumours. BMC Cancer 8: 2, 2008.

10. de Aguiar A Jr, Kowalski LP and de Almeida OP: Clinicopathological and immunohistochemical evaluation of oral squamous cell carcinoma in patients with early local recurrence. Oral Oncology 43: 593-601, 2007.

11. Parker B and Sukumar S: Distant metastasis in breast cancer: molecular mechanisms and therapeutic targets. Cancer Biol Ther 2: 14-21, 2003.

12. Livak KJ and Schmittgen TD: Analysis of relative gene expression data using real-time quantitative PCR and the 2(-Delta Delta C(T)) method. Methods 25: 402-408, 2001.

13. Young JS, Lumsden CE and Stalker AL: The significance of the tissue pressure of normal testicular and of neoplastic (BrownPearce carcinoma) tissue in the rabbit. J Pathol Bacteriol 62: 313-333, 1950.

14. Basson MD, Yu CF and Herden-Kirchoff O: Effects of increased ambient pressure on colon cancer cell adhesion. J Cell Biochem 78: 47-61, 2000.

15. Thamilselvan V and Basson MD: Pressure activates colon cancer cell adhesion by inside-out focal adhesion complex and actin cytoskeletal signaling. Gastroenterology 126: 8-18, 2004.

16. van der Voort van Zyp J, Thamilselvan V, Walsh M, Polin L and Basson MD: Extracellular pressure stimulates colon cancer cell adhesion in vitro and to surgical wounds by Src (sarcoma protein) activation. Am J Surg 188: 467-473, 2004. 
17. Lazaris AC, Theodoropoulos GE, Davaris PS, Panoussopoulos $\mathrm{P}$ Nakopoulos L, Kittas C and Golematis BC: Heat shock protein 70 and HLA-DR molecules tissue expression. Prognostic implication in colorectal cancer. Dis Colon Rectum 38: 739-745, 1995.

18. Lazaris AC, Chatzigianni EB, Panoussopoulos D, Tzimas GN, Davaris PS and Golematis BC: Proliferating cell nuclear antigen and heat shock protein 70 immunolocalization in invasive ductal breast cancer not otherwise specified. Breast Cancer Res Treat 43: 43-51, 1997.

19. Bauer K, Nitsche U, Slotta-Huspenina J, et al: High HSP27 and HSP70 expression levels are independent adverse prognostic factors in primary resected colon cancer. Cell Oncol (Dordr) 35 197-205, 2012.

20. Quintayo MA, Munro AF, Thomas J, et al: GSK3 $\beta$ and cyclin D1 expression predicts outcome in early breast cancer patients Breast Cancer Res Treat 136: 161-168, 2012.

21. Shih LC, Tsai CW, Tsai MH, et al: Association of cyclin D1 genotypes with nasopharyngeal carcinoma risk. Anticancer Res 32: 1093-1098, 2012.

22. Huang SF, Cheng SD, Chuang WY, et al: Cyclin D1 overexpression and poor clinical outcomes in Taiwanese oral cavity squamous cell carcinoma. World J Surg Oncol 10: 40, 2012.
23. Polyak K, Kato JY, Solomon MJ, et al: p27Kip1, a cyclin-Cdk inhibitor, links transforming growth factor-beta and contact inhibition to cell cycle arrest. Genes Dev 8: 9-22, 1994.

24. Sherr CJ and Roberts JM: CDK inhibitors: positive and negative regulators of G1-phase progression. Genes Dev 13: 1501-1512, 1999.

25. Moeller BJ, Richardson RA and Dewhirst MW: Hypoxia and radiotherapy: opportunities for improved outcomes in cancer treatment. Cancer Metastasis Rev 26: 241-248, 2007.

26. Cerniglia GJ, Pore N, Tsai JH, et al: Epidermal growth factor receptor inhibition modulates the microenvironment by vascular normalization to improve chemotherapy and radiotherapy efficacy. PLoS One 4: 8, 2009.

27. Liao D and Johnson RS: Hypoxia: a key regulator of angiogenesis in cancer. Cancer Metastasis Rev 26: 281-290, 2007.

28. Carmeliet P: Angiogenesis in health and disease. Nat Med 9: 653-660, 2003.

29. Mizobuchi H, García-Castellano JM, Philip S, et al: Hypoxia markers in human osteosarcoma: an exploratory study. Clin Orthop Relat Res 466: 2052-2059, 2008. 